\title{
A Galactic Example of a Massive Chimney
}

\author{
N. M. McClure--Griffiths \\ Australia Telescope National Facility, CSIRO, PO Box 76, Epping NSW \\ 1710, Australia \\ J. M. Dickey \\ Department of Astronomy, University of Minnesota, 116 Church St SE, \\ Minneapolis, MN 55455, USA \\ B. M. Gaensler \\ Harvard-Smithsonian Center for Astrophysics, 60 Garden St MS-6, \\ Cambridge, MA 02138, USA
}

\section{A. J. Green \\ School of Physics, Sydney University A28, NSW 2006, Australia}

\begin{abstract}
One mechanism for spreading hot, metal enriched gas away from galaxies is through gigantic chimneys formed in the disk of a galaxy. Chimneys form when shells or bubbles blown by many massive stellar winds and supernova explosions grow large enough to exceed the neutral hydrogen ( $\mathrm{H} \mathrm{I}$ ) scale height of the disk. The shells then become unstable at their polar regions and expand rapidly, breaking out to the galaxy's halo. If galactic fountain models are correct the hot gas liberated by these chimneys should cool into H I cloudlets high above the galaxy's disk. The Milky Way provides the nearest laboratory to search for these objects in order to study how they form and the fate of the expelled gas. While we expect tens of chimneys in the Milky Way to account for the thermal support of the halo there are only a few known chimneys. Here we present an H I study of one Galactic chimney GSH $277+00+36$. GSH $277+00+36$ is the the only chimney known to have blown out of both sides of a galactic disk. We discuss the development of RayleighTaylor instabilities in this object and the role those may have had in the formation of the chimney.
\end{abstract}

\section{Introduction}

Neutral hydrogen (H I) shells, as a class of objects, have a significant impact on the structure and dynamics of the interstellar medium. These objects are usually detected as voids in the $\mathrm{H}$ I, with walls of swept-up material. They range in size from tens of parsecs to kiloparsecs and are often the largest discrete objects observed in galaxies. It is believed that most shells were formed from 
supernovae, stellar winds and the combined effects of both. The largest of the $\mathrm{H}$ I shells have formation energies of $10^{52}-10^{53} \mathrm{ergs}$, requiring $10-100$ massive stars if they are the product of stellar winds and supernovae. These can grow large enough to exceed the scale height of the $\mathrm{H}$ I disk and expand unimpeded into the Galactic halo, creating a "chimney". Chimneys are an important source of metals and thermal support for the Galactic halo. However, there are very few known chimneys in the Milky Way, with some prominent examples being the W44 chimney (Normandeau, Taylor, \& Dewdney 1996), the Stockert Chimney (Müller, Wennmacher, \& Reif 1989), and the Scutum supershell (Callaway et al. 2000).

Unfortunately there are few observations of $\mathrm{H}$ I chimneys that allow us to study their detailed physics, in particular their interaction with the surrounding interstellar medium (ISM) and the process of chimney break-out. Most observational studies of $\mathrm{H}$ I chimneys are limited by images with low spatial resolution. We know from recent hydrodynamic simulations of $\mathrm{H}$ I shells and chimneys (e.g. de Avillez \& Berry 2001; de Avillez \& Breitschwerdt 2003) that these objects should have a wealth of complicated structure with resolved features on the scale of a few parsecs. The simulations reveal hydrodynamic instabilities that have a significant impact on $\mathrm{H}$ I shell evolution, including chimney production (e.g. Mac Low, McCray, \& Norman 1988; Breitschwerdt, Freyberg, \& Egger 2000; de Avillez \& Berry 2001). As a large H I shell evolves the dense walls begin to develop Rayleigh-Taylor instabilities, which can lead to the fragmentation and cloud production along the shell (Mac Low \& McCray 1988; de Avillez 2000). In addition, as shells reach sizes comparable to the $\mathrm{H}$ I disk scale height they become Rayleigh-Taylor unstable along their polar caps, which can break the shell and create a chimney. To test the numerical models of $\mathrm{H}$ I shell evolution, high resolution images of large $\mathrm{H}$ I shells are required. The Milky Way is an ideal environment in which to study the instability processes that effect chimney creation. We can observe Galactic chimneys with parsec-scale resolution and examine their detailed physics. Here we present a high-resolution study of a Galactic chimney. We discuss the presence of gas instabilities along the shell walls.

\section{GSH 277+00+36: A Case Study}

GSH $277+00+36$ was discovered in the low resolution Parkes component of the SGPS (McClure-Griffiths et al. 2000). The chimney appears as an H I void centered in the Galactic plane with multi-channel chimneys extending far above and below the plane. This chimney is located at the edge of the SagittariusCarina spiral arm at a kinematic distance of $6.5 \pm 0.9 \mathrm{kpc}$. The main portion of the GSH $277+00+36$ shell is centered on $l=277^{\circ}, b=0^{\circ}, v=+36 \mathrm{~km} \mathrm{~s}^{-1}$ and extends over approximately six degrees of longitude. The void is apparent over a wide range of LSR velocities, from $v \sim 15 \mathrm{~km} \mathrm{~s}^{-1}$ to $v \sim 55 \mathrm{~km} \mathrm{~s}^{-1}$. The wide velocity width suggests that the shell is still expanding with a velocity. $v_{\text {exp }}$, of $20 \mathrm{~km} \mathrm{~s}^{-1}$. The chimney's vertical channels do not appear bounded at the extremes and span more than ten degrees both above and below the Galactic mid-plane. Assuming a distance of $6.5 \mathrm{kpc}$, the main portion of the shell has a diameter of $610 \pm 90 \mathrm{pc}$, and the chimney extensions reach more than 
$1 \mathrm{kpc}$ above the mid-plane. The shell is quite massive with a swept up mass of $M_{\text {swept }}=(2.7-5.6) \times 10^{6} \mathrm{M}_{\odot}$. McClure-Griffiths et al. (2000) estimate that the equivalent energy initially required at the center of the shell to account for the shell's current size and expansion is $\sim 2.4 \times 10^{53} \mathrm{erg}$. This implies that if the shell were produced by stellar winds and supernovae, several hundred massive stars must have existed within the shell. However, the exact formation mechanism for the shell is unclear. McClure-Griffiths et al. (2000) discuss and rule-out several possibilities, including the impact of a high-velocity cloud with the Galactic disk. Because of the shell's distance, no detection of any remaining cluster of $\mathrm{O}$ and B stars was possible. In addition, McClure-Griffiths et al. (2000) detect no X-ray, infrared or radio continuum emission associated with the shell. Without direct evidence for the formation of the shell, it is difficult to place limits on the age of the shell. However, those authors estimated an upper limit to the age of $\sim 20$ Myr based on the observation that the shell has not experienced significant shearing due to differential rotation in the Galaxy.

\subsection{Small-scale structure in GSH $277+00+36$}

Using the Australia Telescope Compact Array and the Parkes Radiotelescope we have imaged the $\mathrm{H}$ I associated with GSH $277+00+36$ at 3 arcminute resolution. At the kinematic distance of this shell, the spatial resolution of the images is 5.6 pc. These data were presented in McClure-Griffiths et al. (2003). We refer the reader to that paper for a more complete analysis. In Figure 1 we show an $\mathrm{H}$ I image of the chimney near the central LSR velocity of $v=36 \mathrm{~km} \mathrm{~s}^{-1}$. The figure shows many small structures, $\sim 6-10 \mathrm{pc}$, at the edges of the shell.

\subsection{Gas Instabilities}

Hydrodynamic simulations suggest that gas instabilities, particularly RayleighTaylor (R-T) instabilities, have a significant effect on the evolution of $\mathrm{H}$ I shells, chimney production, and even the dissolution of the shell. For instance, we believe that chimneys are formed once the shell size becomes comparable to the scale height of the H I disk. Simulations by de Avillez (2000) and de Avillez $\&$ Berry (2001) show that the density gradient towards the halo provides a $z$ acceleration which is R-T unstable when coupled with the existence of a dense wall above a tenuous interior. Depending upon the position of the shell with respect to the mid-plane, the upper and/or lower caps of the expanding shell will eventually break-out to form a chimney with narrow conduits on both sides of the plane.

In GSH $277+00+36$ there are many structures that may be due to RayleighTaylor (R-T) instabilities. The most striking are the channels forming the chimney of GSH $277+00+36$. As noted by McClure-Griffiths et al. (2000) and seen in Figure 1, GSH $277+00+36$ is not a cohesive, conical out-flowing chimney like the W44 chimney (Normandeau, Taylor, \& Dewdney 1996). Whereas the W44 chimney extends as a cone up from the OB association at its base, GSH $277+00+36$ appears to have broken through the $\mathrm{H}$ I disk layer in at least two different places, above and below. In this characteristic GSH $277+00+26$ agrees well with de Avillez \& Berry (2000) and de Avillez (2001) simulations. In these simulations the largest supershells are observed to break up with hot gas escaping via relatively narrow channels to the upper disk. 


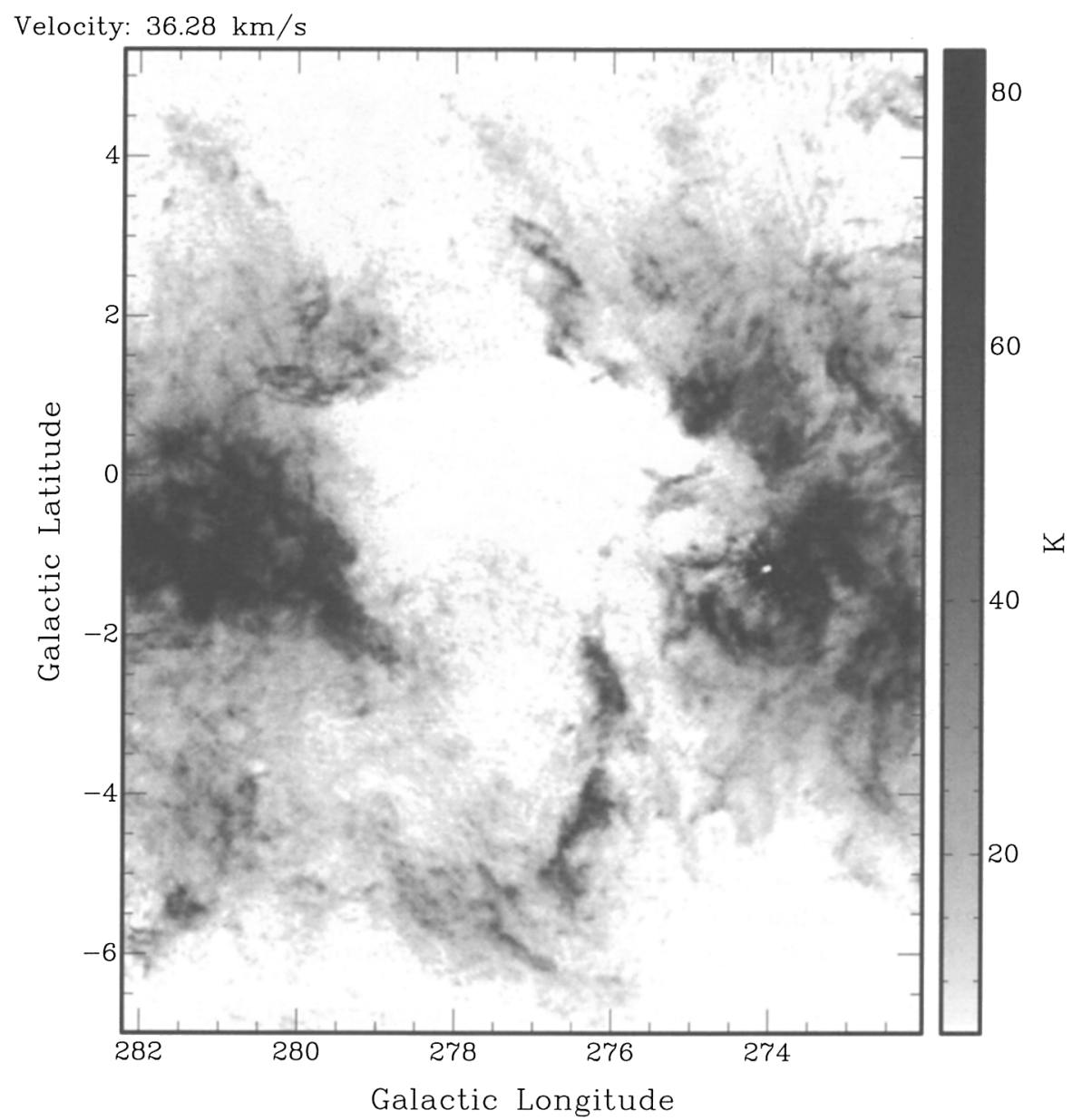

Figure 1. H I image of Galactic chimney GSH $277+00+36$ at 3 arcminute resolution made with the Australia Telescope Compact Array and the Parkes Radiotelescope (McClure-Griffiths et al. 2003). The grey-scale is linear and goes from $0-85 \mathrm{~K}$ as shown in the wedge at the right. The chimney is formed by the two conduits at the top and the bottom of the main $\mathrm{H}$ I void.

Another example of a possible instability formed structure occurs at velocities around $v=40 \mathrm{~km} \mathrm{~s}^{-1}$ where the shell wall appears scalloped with regular periodicity. Figure 2 is an image of the main area of GSH $277+00+36$ at $v=40.4 \mathrm{~km} \mathrm{~s}^{-1}$. The scalloping of the edge is most apparent on the left-hand side of the shell. The scallop manifests itself as a series of arcs whose cusps point into the shell. The cusps of the arcs are separated by $\sim 100$ pc. These are reminiscent of arcs seen along the shell wall in the simulation of Tomisaka (1998). We suggest that these, too, may be the product of a R-T instability at the shell wall. In this case, the cusps of the arcs are the beginnings of R-T fin- 


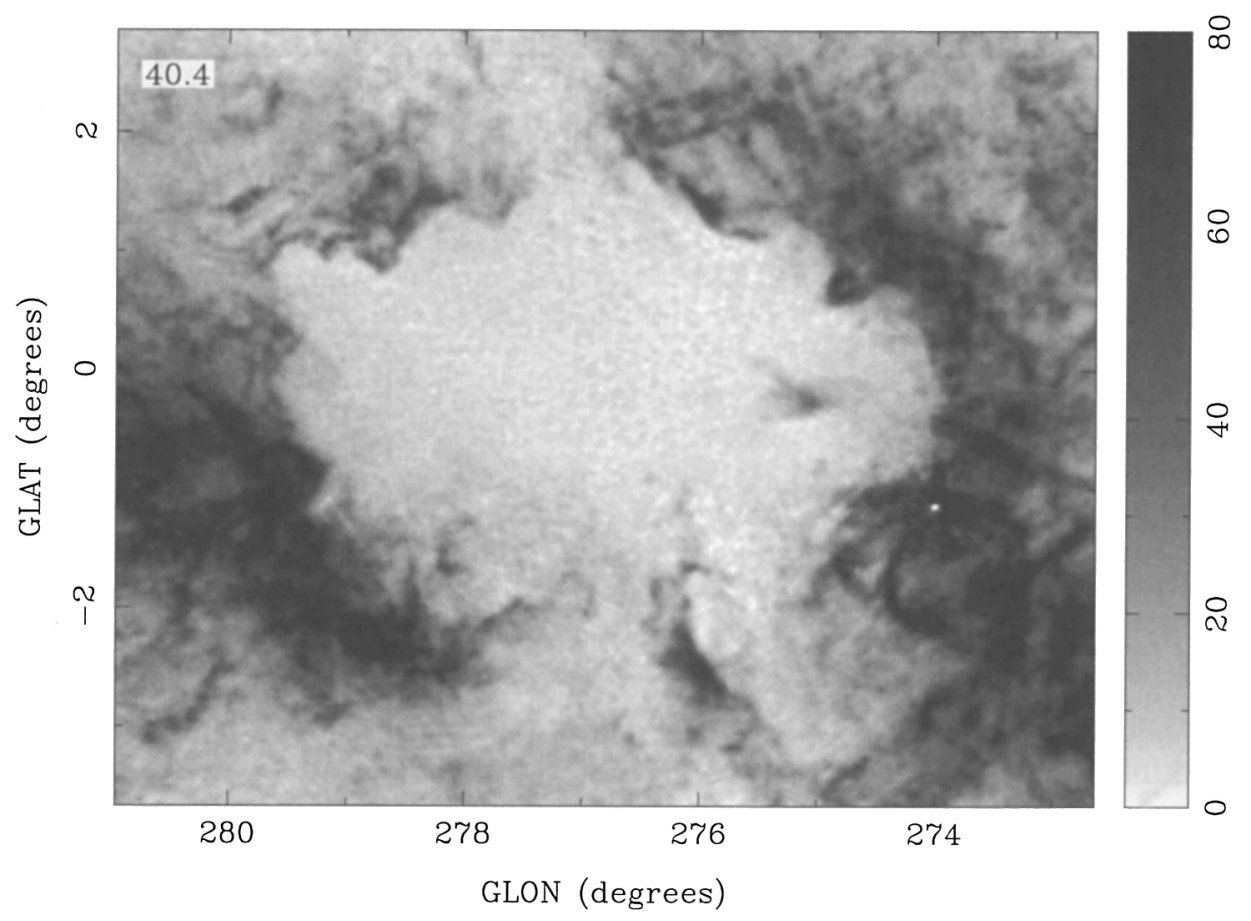

Figure 2. Grey scale image of GSH $277+00+36$ at $v=40.4 \mathrm{~km} \mathrm{~s}^{-1}$. The greyscale is linear from 0 to $80 \mathrm{~K}$, as shown at the wedge on the right. Notice the scalloping of the shell wall all around the shell.

gers "dripping" into lighter material in the shell interior. In the shell exterior we would expect to see the classic R-T caps familiar in hydrodynamic simulations (e.g. Mac Low \& McCray 1989). The morphology of the loop at the upper, left corner of the shell is consistent with R-T caps.

In McClure-Griffiths et al. (2003) we compare these instabilities with analytic solutions for the growth of R-T instabilities in the presence of magnetic fields. Given assumed densities for the shell wall and interior gas, R-T instabilities developing in a uniform magnetic field will have a minimum, fastest growing wavelength. These observations could resolve $\mathrm{R}-\mathrm{T}$ wavelengths of $<10 \mathrm{pc}$. However, the smallest wavelength of R-T instabilities that we observe is $\sim 20 \mathrm{pc}$. Assuming a typical magnetic field strength on the order of $\sim 3 \mu \mathrm{G}$ and an interior to exterior density contrast of $n_{2}=(10-20) n_{1}$, where $n_{1} \sim 0.05 \mathrm{~cm}^{-3}$, we found that the minimum wavelength is on the order of $15-30 \mathrm{pc}$. The agreement between the analytic R-T properties and those of the observed smallscale structures suggests that these structures may be magnetically confined R-T instabilities. 


\section{Conclusions}

Studies of H I chimneys in the Milky Way offer us a rare opportunity to examine the gas instability processes that effect chimney production. In recent years hydrodynamic simulations of these objects have advanced sufficiently to show in detail the development of Rayleigh-Taylor instabilities on size scales ranging from parsecs to hundreds of parsecs. Simulations show that chimneys may form with narrow conduits as Rayleigh-Taylor instabilities break the polar regions of a shell. GSH $277+00+36$ appears to agree very well with these simulations, revealing four narrow chimney conduits to the halo. There is further evidence for Rayleigh-Taylor instabilities along the shell walls, which have a scalloped appearance. We show that size of the smallest of these instabilities agrees well with magnetically confined Rayleigh-Taylor instabilities.

Though many chimneys are required to provide thermal support for the Galactic halo, only a few examples are known. Unfortunately, significant selection effects make it difficult to find chimneys in the Milky Way. Those chimneys that we do know, however, are ideal for exploring the detailed physics of these complicated objects.

\section{References}

Breitschwerdt, D., Freyberg, M. J., \& Egger, R. 2000, A\&A, 361, 303

Callaway, M. B., Savage, B. D., Benjamin, R. A., Haffner, L. M., \& Tufte, S. L. 2000, ApJ, 532, 943

de Avillez, M. A. 2000, MNRAS, 315, 479

de Avillez, M. A. \& Berry, D. L. 2001, MNRAS, 328, 708

de Avillez, M. A. \& Breitschwerdt, D. 2003, Revista Mexicana de Astronomia y Astrofisica Conference Series, 15, 299

Mac Low, M. \& McCray, R. 1988, ApJ, 324, 776

Mac Low, M., McCray, R., \& Norman, M. L. 1989, ApJ, 337, 141

McClure-Griffiths, N. M., Dickey, J. M., Gaensler, B. M., \& Green, A. J. 2002, ApJ, 578, 176

McClure-Griffiths, N. M., Dickey, J. M., Gaensler, B. M., \& Green, A. J. 2002, ApJ, 594, 833

Müller, P., Wennmacher, A., \& Reif, K. 1989, Astronomische Gesellschaft Abstract Series, 3, 98

Normandeau, M., Taylor, A. R., \& Dewdney, P. E. 1996, Nature, 380, 687

Tomisaka, K. 1998, MNRAS, 298, 797 